\title{
Frontières
}

\section{Catherine Cyssau (dir. publ.), L'entretien en clinique, Paris, In Press Éditions, 1998, 345 pages}

\section{Luce Des Aulniers}

Volume 12, numéro 2, printemps 2000

URI : https://id.erudit.org/iderudit/1074409ar

DOI : https://doi.org/10.7202/1074409ar

Aller au sommaire du numéro

Éditeur(s)

Université du Québec à Montréal

ISSN

1180-3479 (imprimé)

1916-0976 (numérique)

Découvrir la revue

Citer ce compte rendu

Des Aulniers, L. (2000). Compte rendu de [Catherine Cyssau (dir. publ.), L'entretien en clinique, Paris, In Press Éditions, 1998, 345 pages]. Frontières, 12(2), 86-88. https://doi.org/10.7202/1074409ar

Ce document est protégé par la loi sur le droit d'auteur. L'utilisation des services d'Érudit (y compris la reproduction) est assujettie à sa politique d'utilisation que vous pouvez consulter en ligne.

https://apropos.erudit.org/fr/usagers/politique-dutilisation/
Cet article est diffusé et préservé par Érudit.

Érudit est un consortium interuniversitaire sans but lucratif composé de l'Université de Montréal, l'Université Laval et l'Université du Québec à Montréal. Il a pour mission la promotion et la valorisation de la recherche. https://www.erudit.org/fr/ 
qu'ils n'avaient nulle part pignon sur rue, aucune unité de rattachement n'a pu les protéger des coupures et leur assurer les ressources financières, secrétariales et surtout professorales sans quoi on ne peut maintenir en vie un programme majeur d'études et produire une revue de qualité.

Par une soirée de gala avec piano, danse et chants d'opéra sur un thème "À la frontière des sortilèges" le samedi 31 octobre 1998, la revue Frontières a célébré son dixième anniversaire d'existence. À quels autres sortilèges eût-il fallu recourir pour assurer son développement au cours d'une deuxième décennie?

\section{Notes}

1 La majeure partie de cet article, consacrée à l'analyse de contenu proprement dite, a été rédigée à l'automne 1999. C'est en juin 2000 qu'on a complété l'analyse des évaluations, l'examen de la gestion administrative, technique et financière et la rédaction de l'épilogue.

2 N.D.L.R.: Frontières se présente maintenant comme une "revue québécoise d'information, de recherche et de transfert de connaissances en études sur la mort». Sans effectivement que ce changement de sous-titre ait introduit une modification majeure, il reste que l'exigence à la fois de scientificité et d'accessibilité des textes s'est accrue.

3 N.D.L.R.: d'évidence, il ne s'agira pas ici d'une analyse de contenu telle qu'on l'entend en recherche qualitative, portant sur des récits littéraires, de vie, de pratique, etc. Ainsi, par exemple, une analyse des sous-thèmes traités dans chaque numéro est impossible; toutefois le terme "analyse de contenu» est accrédité pour le type de description ici présenté.

4 N.D.L.R.: la direction de la revue veille au maintien de la qualité scientifique et à l'orientation de la revue, coordonne et anime le travail des équipes et individus, assure les relations publiques, les consultations, les demandes de subvention, la gestion du personnel, etc. ; la direction assume in finale les décisions sur la publication et travaille étroitement avec la rédaction et les responsables tout au long du numéro ; la rédaction, toujours sous la responsabilité de la direction, travaille directement sur le contenu de la revue et plus particulièrement l'évolution des textes et des rapports avec les auteurs. Rédaction et direction sont présentes lors de la phase de production.

5 N.D.L.R.: précision: devant le vacuum effectivement fort périlleux pour la revue, il allait de soi qu'une professeure membre du Comité de rédaction prenne le relais à la direction, de son plein gré.

6 N.D.L.R.: un organisme subventionnaire externe prévoit ce dégrèvement: il arrive que les revues l'emploient autrement, par obligation, ce qui fut parfois le cas. Un dégrèvement d'enseignement fut attribué de manière exceptionnelle par l'UQAM, en 1997.

7 N.D.L.R.: dans les faits, il s'agit des deux numéros du volume 13 , déjà avancés.

\section{Catherine Cyssau (dir. publ.) L'entretien en clinique}

Paris, In Press Éditions, 1998, 345 pages.

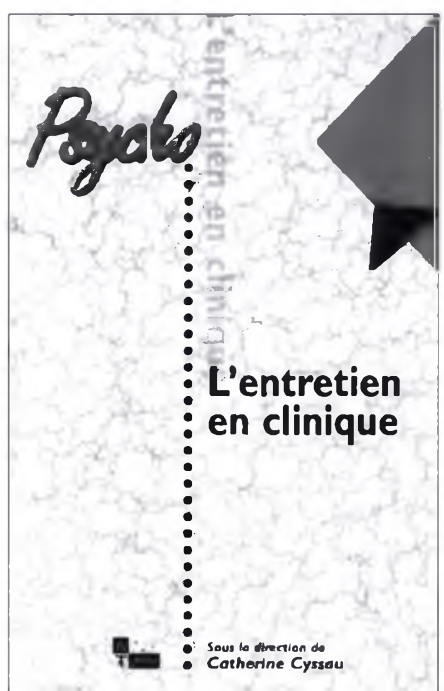

Le terme "entretien» connaît bien des infortunes du fait qu'il soit devenu un mot-valise. Ici, il est sondé avec une rigueur remarquable sous la multitude de prismes de la clinique du psychopathologique. C'est donc tout à fait bienvenu dans l'examen du phénomène de la peur, serti d'angoisse. Or, trois critères sont apposés et se répercutent au long des quelques vingt-deux courts et bien corsés chapitres de l'ouvrage (avec tout autant de collaborateurs): 1) Quels sont les modèles ou présupposés théoriques influençant la pratique de l'entretien?; 2) Quelles sont les modalités (parfois successives) visées par l'entretien?; 3) À la croisée de l'intrapsychique et de l'intersubjectif, quelles sont les formations psychiques à l'œuvre pour le patient, et à quels types d'interactions donnent-elles lieu? C'est en tenant compte de ces trois énoncés que le clinicien tourne le dos à la technique-prêt-à-porter, "qui sied mal à la parole dès lors qu'elle véhicule, avec tout le reste de l'expression et le mode de présence physique, émotionnelle, du patient à l'entretien, les processus psychiques et l'individuation symptomatique propre à chacun.» (p. 15).
Et là, les gourmets peuvent se mettre à table. Mais comment donner une idée fidèle du menu? Puisque chaque auteur admet d'emblée la complémentarité, voire l'imbrication des modèles de référence, le tout généralement transmis avec une grande clarté, ces aspects ne seront pas répétés. Plutôt que de présenter un survol global, on présentera, mi-arbitrairement, mi-«en questionnements réflexifs» ( $p$. 15) quelques extraits de chaque chapitre, dans la mire du thème de ce numéro de Frontières, concentrés alors plus en première partie.

1- Perspectives théoriques

"La parole et le langage en psychanalysen: toute mention d'un malaise serait sous-tendue par la croyance en la toute-puissance des idées, ainsi qu'en celle de l'interlocuteur, rêvant à l'éternité de l'inscription comme de la résolution. «ll semble que la transmutation liée au surgissement d'un sens imprévisible soit d'autant plus féconde que la parole est d'emblée reçue dans la stratification de ses dimensions: plainte corporelle, séduction, référence à un principe de légalité, récit établissant un passé, autant de niveaux s'offrant à être subvertis par l'effet d'une rencontre verbale. L'effet de sens interviendra nécessairement en prenant le visage de l'hôte indésirable ou du moins inattendu.» (p. 32).

"Les modèles de communication psychanalytique" renvoient quant à eux aux champs intercroisés de l'intrapsychique - plus à distance de l'expérience - et de l'interpersonnel. S'appuyant I'un sur l'autre, ils donnent lieu à des modèles qui sont ici saisis dans la différence entre cure et entretien. Au terme de ces modèles, apparaît la clinique du narcissisme dont «le caractère polysémique du terme (narcissisme primaire et secondaire, phallique, délibidinalisation, etc.) risque d'en faire également un concept passe-partout. II sera toujours possible de parler d'une inflation ou d'une faille narcissique. Plus intéressant sans doute est le souci d'explorer l'aspect négatif du narcissisme, c'est-à-dire la pauvreté des investissement libidinaux que l'on peut assez faci- 
lement repérer lors de l'entretien." (p. 44).

"L'entretien psychologique" élabore les règles de l'échange d'après l'apport de Rogers et de la pragmatique, tout en prenant des libertés avec ces règles.

"L'entretien psychiatrique", quoique plus excentrique dans cette galerie, du fait des idéaux scientifiques en termes de diagnostic et de conduite à suivre, précise notamment le risque "de développer une conception organiciste de la pathologie mentale et plus encore le risque d'apporter aux pathologies psychiques le même type de réponse qu'aux pathologies somatiques, c'est-à-dire essentiellement chimiothérapique" (p. 60). C'est de cette distinction que cet entretien trouve sa spécificité.

Tout entretien peut procéder selon "la perspective phénoménologique» dans la mesure où, a prio$r i$, il distingue connaissance objective et expérience phénoménale, cette dernière décrivant et spécifiant la manière dont les choses nous apparaissent. S'éloignant d'une acception courante dans le sens du mode d'apparaître, observé par le clinicien, on privilégie ici l'acception philosophique de ce qui apparaît au patient. Dans la lignée de Husserl, il s'agit de faire décrire cette expérience et de comprendre comment l'objet y est appréhendé. L'intentionnalité ou le projet tient dans la conscience perceptive, celle du corps, du temps et d'autrui. Les altérations de l'une et l'autre entraînent des pathologies, telle celle dans le rapport avec autrui (intersubjectivité): "Le psychotique semble avoir perdu cette "compétence" d'intersubjectivité quand il élabore son monde délirant." (p. 69). En dépit de l'actualité de cette perspective, il reste à travailler la reconnaissance et l'articulation de la phénoménologie en psychopathologie.

«Force est de constater le poids de la culture dans les organisations identitaires des individus et son influence dans les rapports qu'ils entretiennent", ainsi s'amorce "Transculturel: I'ailleurs et l'ici». Culture d'origine et culture d'accueil, renvoient l'une et l'autre à ce qui est dissimulé ou proclamé dans l'expérience culturelle du trouble psychique. À ce domaine expérientiel s'ajoutent le redoublement de l'étrangeté, dans les cultures forcément différentes du clinicien et de la personne souffrante, puis les inscriptions culturelles intemporelles (ayant trait aux définitions du familier et de l'étranger, notamment). De ces trois paradigmes émerge un lieu de passage entre deux mondes: "L'entretien clinique sensible aux entrelacs du psychique et du culturel aura rempli sa tâche s'il offre la possibilité de renoncer à la nostalgie de l'origine pour accéder à la restitution des origines." (p. 77)

Puisque les performances du langage, du raisonnement, de la perception, de la mémoire et le contrôle moteur sont parfois altérés, "la perspective cognitive» ou le cognitivisme en psychopathologie se trouve d'emblée plurielles, à des niveaux fort hétérogènes du fonctionnement mental ou cérébral. Les divers niveaux d'investigation (neurophysiologie, psychologie, linguistique) ont toutefois en commun des modèles logico-mathématiques ainsi que des exigences de validation qui tendent à faire "naturaliser» langage, mémoire, jusqu'à la pensée. En clinique, on peut associer non pas des processus, mais des contenus de pensée (croyances, représentations) à des ètats précis. Ces "cognitions", influant sur les comportements, sont traitées au plan du symptôme, plutôt qu'en considérant les conditions de leur genèse. L'altération cognitive peut être étudiée en termes d'interactions entre émotions et processus cognitifs. Ainsi «ont été particulièrement étudiés les effets de l'anxiété sur les fonctions cognitives élémentaires et a été mise en évidence la prévalence de ces effets sur les processus attentionnels." (p. 83). Mais l'inverse peut être tout aussi juste. De plus, on ne peut ramener des manifestations pathologiques à des altérations précises. Comme il est difficile d'observer ces troubles en entretien, on a recours à une évaluation quantitative. Enfin, la fécondité de cette approche tient en bonne partie à son effort d'articulation interdisciplinaire.

Influencée par les travaux de G. Bateson, la perspective écosys- témique en entretien situe la pathologie mentale dans les contextes de communication, donc au niveau interpsychique. Le patient est symptôme. Comme la famille en est le lieu d'apprentis sage et que son implication émotionnelle est d'autant augmentée par la dispensation des soins, moins forte, c'est au plan des psychosociopathies (délinquance, violence, relations d'emprise, de maltraitance) que l'entretien de cet ordre est susceptible de réussir: par le changement (ou la crise qu'il provoque). Le "comment" requiert des aptitudes particulières du thérapeute, lequel agit souvent à plusieurs. Le "pour quoi faire" se lit ici dans une éthique du choix par les protagonistes, dans l'épistémologie de la double description (au sens de propriété de chacun des deux individus et dépassant cette dualité), et enfin dans une modulation contextuelle des subjectivités et intersubjectivités.

\section{II- Entretiens d'investigation}

Cette partie de l'ouvrage dégage quatre types d'entretiens: celui de recherche traitera différemment le prélèvement du contenu (les préalables, les propriétés, les formes, l'analyse sont admirablement cernés), tout en n'ignorant pas les inconnues et donc les risques, de même que les implications déontologiques, éthiques et juridiques. Le défi reste de se tenir entre d'un côté, la soumission au discours du sujet, la difficile généralisation et de l'autre, une systématisation appauvrissante. $\dot{A}$ cet égard, les récents progrès en méthodologie qualitative sont éclairants.

L'entretien diagnostic se réfère à des fonctions (soi, narcissisme, identification, transfert, contretransfert...) perceptibles en une ou plusieurs rencontres; sont soulignées la difficulté de l'objectivité en psychiatrie, la différence phénoménologique entre la névrose et la psychose et les variétés du transfert et du narcissisme, en concluant sur la limite de la clinique diagnostique: le patient état-limite.

"L'entretien autour des tests projectifs", s'établissant en contexte institutionnel, auprès de sujets aux prises avec de graves difficultés, aborde les questions des rencontres préalables et post-tests, de même que la restitution, en insistant sur la qualité relationnelle.

Domaine moins documenté, l'entretien en clinique expertale renvoie plus désormais au psychologique, avec cette particularité que dans la plupart des cas, il n'est pas demandé par le sujet; les implications complexes sont développées (à propos du cadre et du regard de l'expert, de son statut, de la sensibilité du sujet) de même que l'origine de la clinique, reliée à l'indépendance de l'expert.

\section{III- Entretiens psychothéra-} piques

"Le premier entretien" puise à deux modèles théoriques engageant un rapport spécifique à la technique analytique, celui de préliminaire chez freud et celui de Winnicott. À tout le moins, "le danger nommé [par le patient] rend la vie moins menaçante." (p. 152).

Dans "le modèle de la névrose" se délimitent le cadre et la souple définition toujours, sa portée, ainsi que: de l'anamnèse autobiographique à la réminiscence; du factuel à l'interprétable; de la démonstration de l'affect à l'émergence de mots; de l'inhibition dépressive à l'investissement du pensable.

"Névrose traumatique, névrose actuelle du comportement, "névrose» de somatisation" rendent compte d'une sensibilité singulière du patient à son destin mortel, qui le désorganisent et l'angoissent. Sont questionnées très précisément la métapsychologie et les aménagements de la rencontre.

Sont de la même manière sondées les complexités des entretiens composant avec "la zone psychotique de la psyché", "les pathologies de l'agir" ou "le paradigme de l'autisme: face aux conduites de retrait, d'autosuffisance et à la dimension du non-verbal.»

IV- Variantes cliniques

Elles concernent les victimes de traumatismes, le milieu judiciaire, les consultations avec le couple et la famille, autour du nourrisson, se consacrant également à la question "du discours sur l'enfant au discours de l'enfant" et à la dyna- 
mique à l'adolescence, et certainement, avec la personne âgée"

V- Le clinicien face à l'entretien: institution, transmission, formation"

Ce chapitre offert en conclusion souligne notamment "qu'il est temps de dépasser la sous-traitance du psychologique par le psychiatrique, ou son inversion, qui conduisent, par trop souvent, à l'incommunicabilité institutionnelle du psychopathologique." (p. 324). L'un et l'autre peuvent autant être consultants que psychothérapeutes et bien plus, la prise en charge peut être double, le premier ètant acteur au niveau de la réalité concrète du patient, le second, au niveau de la réalité psychique. Cette compatibilité articulée permet en outre d'assouplir les inévitables réactivités des équipes soignantes, éducatives ou sociales. Enfin, sont traitées les voies de transmission, tels l'écrit et le vidéo, et la supervision.

Il est rare que l'on puisse disposer d'un ouvrage qui redéploie les concepts fondamentaux tout en traitant avec grande finesse des questions très contemporaines de pratiques. Celui-ci est en ce sens complet et à la portée autant d'etudiants que de cliniciens et offre peut-être bien à tout patient une forme de mise à distance de ce qui l'afflige.

\section{Luce Des Aulniers}

\section{François Dagonet, Tobie Nathan}

\section{La mort}

\section{vue autrement}

Le Plessis-Robinson, Institut Synthélabo pour le progrès de la connaissance, 1999, 141 pages.

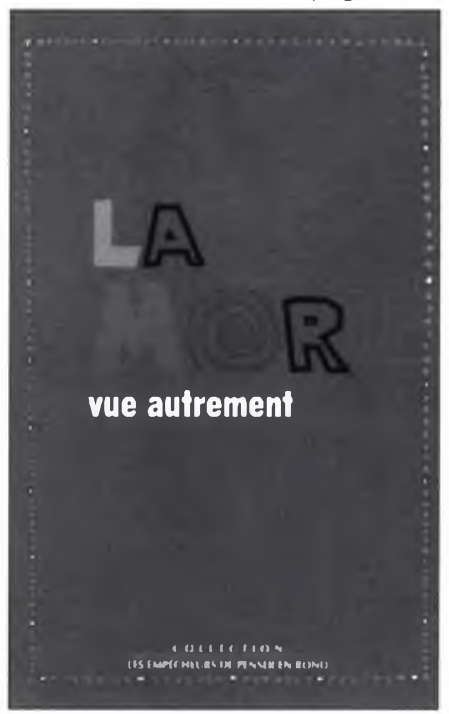

On connait le travail interdisciplinaire (ethnopsychiatrie) de Tobie
Nathan, un de ceux qui résiste au nivellement que cette tendance méthodologico-épistémologique actuelle n'avait pas prévu; dans son cas, parlons plutôt d'hybridation féconde, en ceci que l'ailleurs n'est pas folklorisé, que l'Occident du nord n'est pas le centre du monde, avec comme résultat des propositions de pensée liantes plutôt que des vérités systématiquement assénées. En ce sens, il est difficile de comprendre que son apport ne tienne ici que dans une trentaine de pages, articulant par ailleurs des pans de ses travaux antérieurs, sous le titre "Le mort et son représentant». En voici une proposition: "La mort étant la disparition soudaine d'un être, expulsè du monde où il se trouvait, la mort est donc ce qui me contraint à donner naissance à d'autres êtres." (p. 106). Dès lors, "C'est du fait de leur changement de monde qu'ils [les morts] contraignent les survivants à adopter des attitudes, des comportements, à obéir à des règles qui n'appartiennent pas au monde ordinaire, comme s'ils signifiaient qu'ils s'adresseraient désormais aux humains à partir d'un autre lieu." (p. 119). Si les morts agissent de ce lieu, parfois en désordre, l'autorité du chef du groupe, de l'État, ou encore la dramaturgie notariale, les représentera. Cette contrainte sur les vivants est donc groupale et ritualisée (les deux, inséparables): c'est le groupe et le rite qui convoquent savamment - sans s'en douter!! - une vie autre aux morts, cette dernière pérenne et manifeste au quotidien des vivants. Tout destin serait en ce sens imbriqué à la vie du groupe, si bien que le rite de deuil ne peut être accompli en solitaire. Il en va du mort comme de l'impétrant à l'initiation (cette association a aussi été explorée antérieurement par L.-V.. THOMAS): toute existence conduit naturellement à un conglomérat figé, hétéroclite et en partie insignifiant qu'il faut périodiquement et culturellement casser. L'idéal à atteindre par ces fractures multiples tient en l'unicité et en la densité: de rares morts deviennent ancêtres et de rarissimes, divinités. "Nous voyons donc que le cadavre, avec toutes ses métamorphoses (cf. L.-V. Thomas, Le cadavre), est ici le noyau d'une construction conceptuelle particulièrement complexe qui diffuse sa logique dans tous les secteurs de la vie sociale. Un modèle de réflexion s'impose aux vivants, sourd de manière permanente des pratiques rituelles et s'impose comme une logique de l'univers. Selon ce modèle [...], ne pas initier les êtres, c'est se figer dans leurs tendances toujours infructueuses de contenir la multiplicité; les voir se rigidifier dans l'inutile effort d'empêcher l'implosion qui finira nécessairement par les transformer en grains de sable.» (p. 115). II nous faut poursuivre comme lecteurs: nos contemporains veulent pour beaucoup devenir grains de sable, autonomisés qu'ils se croient du socius... Or, être grains de sable, pour l'auteur, c'est être mort doublement. Quid alors du deuil?

L'objet libidinal, construit par Freud et repris dans les theories contemporaines du deuil (comme équivalent à un autre problème psychique) en prend ici pour son rhume: le travail de deuil ne se réaliserait pas dans le sens de réactivation de la perte, mais bien dans celui du réapprentissage de soi, de l'autre, sur soi, sur l'autre. (Qui a décrété que ces deux termes étaient antinomiques?) "Traiter le mort implique toujours le recours aux techniciens locaux, alors que traiter l'endeuillé, en le détachant de ses liens les plus profonds, permet les captures d'âmes nécessaires à la construction des plus grands empires." (p. 127). Se profilent ainsi les chicanes de territoire... qui dépassent les auditoriums pour s'étaler dans les média.

Ceci nous entraîne à évoquer ce qui constitue la large part du document, dans la contribution du philosophe François Dagognet, qui d'ailleurs répond à la fin à son coauteur: on apprend que "peu de penseurs se sont attachés à ce problème, sauf pour le minimiser ou le nier» (p. 13); le premier chapitre résume "ce que la science nous dit", notamment sur la volonté de continuation indéfinie de la vie et surtout sur la biologie de la mort dans la vie, de la vie dans la mort: "la société, à travers ses spécialistes [dont la médecine légale], continue à regarder "le corps" moins comme un "reste" que comme ce qui conserve les marques indélébiles de notre personnalité." (p. 29). On cherchera en vain en quoi: se glisse ici un doute qui persistera, en ceci que la psyché, à la charnière du biopsychique et du culturel, est "oubliée", en dépit d'avancés de ce type: "L'idée juste englobe tout et ne s'en tient pas à un fragment." (p. 19).

Les deux chapitres centraux survolent le système culturel: en premier lieu «il convient de mettre au-dehors ce qui s'est glissé audedans, afin d'éviter tout conditionnement. [...] À les sortir de l'ombre, nous pourrons échapper aux stéréotypes et aux idées convenues; il ne nous restera plus qu'à montrer le chemin (plus authentique et plus droit) qui s'ouvrira.»(p. 36, 38). Avec un très petit corpus d'auteurs sélectionnés, la peur de la mort est décelée dans "la société archaïque [sic], qui se prolonge parmi nous sous formes de rites, de dispositifs folkloriques, de cérémonies obligées, de superstitions" (p. 38) dont la "nocivité» est déclarée. Comme on le dit de la guerre. Cette idée de la mort (comme si, selon l'auteur, la mort n'inspirait que terreur) nous amène à la chasser, dans la personne du mourant et dans un rapport (à noter, jamais ambigu) aux morts qui les rend redoutables. Quant à l'eschatologie, second socle culturel, l'auteur démontre comment le platonicisme est thanatophile: «il supprime ou éteint la vie, tenue pour ce qui nous perd et nous souille." (p. 47). Enfin, "la descente dans le gouffre de la subjectivité, comme son écoute [...] constituent, à nos yeux, le commencement de la psychopathologie.» (p. 49). "À partir du freudisme, "la mort se naturalise" et se revêt d'aspects encore plus sombres; elle nous infiltre; nous n'arrivons pas à nous laver de la faute ou du meurtre dont elle nous accable, en même temps que nous redoutons la violence destructrice de ce qui nous entoure." (p. 53).

La nouvelle philosophie de la mort, "non nocturne" ne la remet pas dans la vie, au cours de la vie, par exemple dans l'Art, qui "ne touche que les esthètes" ( $p .56)$ mais s'adonne à une perspective "communautaire" de sa survenue. Quoi de neuf par rapport à la confiscation historique et une gestion de la mort?) En premier lieu, "celui qui va quitter la vie connaît une épreuve diminutive: n'est-ce pas le moment de la compenser (au moins partiellement) grâce à ceux qui l'entourent et à tout ce qui rappelle son passé (positif), ainsi que les liens toujours vivants avec ceux qui se trouvent à ses côtés? (p. 61). Ce moment devrait être occasion de réjouissances célébrant la cohésion raffermie du groupe par le mort, augurant de sa réorganisation et de sa refondation. (On veut bien, mais le problème réside dans la séquence - un peu rapide - de cette réjouissance, non dialectisée avec la crise et le chagrin.). En second lieu, pour ce qui concerne les obsèques, est reprise la valence de la terre, préférée au feu: "Grâce à ces morts, rassemblés, nous pouvons revenir à nos racines, aux fondateurs ou aux ancêtres. [...] 